\title{
The Endogenous Danger Signal Uric Acid Augments Contact Hypersensitivity Responses in Mice
}

\author{
LanLan Liu $^{a}$ Hiroko Inoue $^{a}$ Hirofumi Nakayama ${ }^{b}$ Rieko Kanno ${ }^{a}$ \\ Masamoto Kanno ${ }^{a}$ \\ Departments of ${ }^{\mathrm{a}}$ Immunology and ${ }^{\mathrm{b}}$ Molecular Pathology, Graduate School of Biomedical Sciences, \\ Hiroshima University, Hiroshima, Japan
}

\section{Key Words}

Uric acid crystals $\cdot$ Danger hypothesis $\cdot$ Contact

hypersensitivity

\begin{abstract}
Objective: The danger hypothesis proposes that the immune system responds not only to foreign antigens but also to damaged cells or tissues. Recently, uric acid crystals (monosodium urate, MSU) from necrotic cell lysates were identified as a danger signal for dendritic cells (DCs). Our aim was to determine whether MSU modulates immune responses in the skin. Method: We analyzed the effect of MSU on trinitrochlorobenzene-induced contact hypersensitivity responses using BALB/c mice administered potassium oxonate, an uricase inhibitor, to prevent MSU degradation. Ear swelling response after elicitation and activation profiles of DCs and T cells in draining lymph nodes after sensitization were assessed. Results: Intradermal administration of MSU augmented the ear swelling response in potassium oxonateadministered mice and enhanced expression of CD86 and CD40 molecules on DCs in the lymph nodes. Activation of DCs was followed by an increase in CD69+ and CD44+ T cells in CD4+ and/or CD8+ subsets in the lymph nodes 4 days after trinitrochlorobenzene sensitization. Conclusion: These observations demonstrate that MSU is an endogenous dan-
\end{abstract}

ger signal, which augments the contact hypersensitivity response in mice. MSU released from damaged skin may act as an endogenous adjuvant to augment immune response.

Copyright $\odot 2007$ S. Karger AG, Basel

\section{Introduction}

Dendritic cells (DCs) are highly specialized professional antigen-presenting cells (APCs), located predominantly in the skin and mucosa, that sense microbes by pattern recognition receptors. Microbial components (pathogen-associated molecular patterns) provide signals that alert our innate immune systems to infection and generate an adaptive immune response [1]. However, pathogen-associated molecular patterns (nonself) are not the only triggers of innate immunity. Innate immunity can also sense abnormal self through endogenous danger signals $[2,3]$. The danger hypothesis proposes that our immune system senses damaged cells and tissues, and responds through the direct activation of APCs such as DCs [4-9]. Besides conventional models of the immune response, which are based on distinguishing self versus nonself, we believe that the danger hypothesis is also important for understanding allergy, inflammation and autoimmune diseases.

\section{KARGER}

Fax +4161306 1234

E-Mail karger@karger.ch

www.karger.com
(C) 2007 S. Karger AG, Basel

$1015-2008 / 07 / 0743-0177 \$ 23.50 / 0$

Accessible online at:

www.karger.com/pat
Dr. Masamoto Kanno

Department of Immunology, Graduate School of Biomedical Sciences

Hiroshima University, 1-2-3 Kasumi, Minami-ku

Hiroshima 734-8551 (Japan)

Tel. +81 82257 5175, Fax +81 82257 5179, E-Mail mkanno@hiroshima-u.ac.jp 
The first self molecules that were shown to serve as danger signals were the heat shock proteins. Several groups have suggested that some mammalian heat shock proteins purified from necrotic cells induce potent activation of DCs and cytotoxic T cells [10]. Intracellular nucleotides like ATP have also been shown to be danger signals. Nucleotides are released from a variety of cells under conditions of hypoxia, ischemia, inflammation or mechanical stress, and the purinergic receptor P2X7 on DCs detects these extracellular nucleotides [11]. Uric acid in necrotic cell lysates has also been identified as a danger signal [12-15]. Shi et al. [13] showed that uric acid crystals (monosodium urate, MSU) activate DCs in vitro and, when coinjected with a particulate antigen, induce CD8+ T cell responses in vivo. Martinon et al. [16] proposed that the NALP3 inflammasome constitutes some of the proximal sensors for MSU-derived danger signals.

Sutterwala et al. [17] further showed that the NALP3 inflammasome, a proposed sensor of MSU-derived danger signals, is essential to establish contact hypersensitivity (CHS) to the hapten trinitrophenylchloride. CHS is a cellular immune response generated after repeated epicutaneous exposure to contact allergens and is a clinically important syndrome known as allergic contact dermatitis. CHS is also an important experimental model of cellmediated immunity $[18,19]$. CHS can be divided into 2 phases: sensitization and elicitation. In the sensitization phase, skin exposure to haptens leads to maturation of skin DCs, epidermal Langerhans cells and other dermal DCs, which transport the haptenized antigen to regional lymph nodes (LNs) where the antigen is presented to $\mathrm{T}$ cells. These antigen-specific T cells become activated and clonally expand. In the elicitation phase, rechallenge with the same hapten at a distant site leads to an inflammatory reaction that peaks within $24 \mathrm{~h}$. During the peak of the response, the cellular infiltrate contains an abundance of immune cells, especially $\mathrm{T}$ cells, natural killer cells, neutrophils and macrophages.

In the present study, we determined whether MSU modulates the CHS response. We report that subcutaneous application of MSU in mice augmented the trinitrochlorobenzene (TNCB)-induced CHS response, enhanced the expression of costimulatory molecules on DCs in draining LNs and led to an increase in the number of activated $\mathrm{T}$ cells. These observations demonstrate that MSU functions as an endogenous danger signal that increases the CHS response in mice. We hypothesize that MSU, when released from damaged cells, may act as a self-adjuvant to augment skin immune responses.

\section{Materials and Methods}

\section{Reagents}

TNCB (2,4,6-trinitro-1-chlorobenzene) was obtained from Tokyo Kasei (Tokyo, Japan). Uric acid was from Sigma-Aldrich (St. Louis, Mo., USA), and potassium oxonate from Acros Organics (Morris Plains, N.J., USA). MSU crystals were prepared by incubating supersaturated uric acid solutions $(4-5 \mathrm{mg} / \mathrm{ml})$ in $0.1 \mathrm{M}$ borate ( $\mathrm{pH} 8.5$ ) at room temperature for $>48 \mathrm{~h}$, followed by washing with alcohol and acetone.

\section{Antibodies}

The following anti-mouse monoclonal antibodies were obtained from BD Pharmingen (San Diego, Calif., USA) and were used for flow cytometry [fluorescent-activated cell sorting (FACS)] analysis: anti-CD11c-APC (HL3), anti-CD11b-FITC or anti-CD11b-PE (M1/70), anti-CD86-PE (GL-1), anti-CD4-FITC or anti-CD4-PE (RM4-5), anti-CD8-APC (53-6.7), anti-CD25FITC (7D4), anti-CD44-PE (IM7), anti-CD69-PE (H1.2F3) and anti-CD16/CD32 (2.4G2). The anti-mouse CD40-FITC (KJ9024) was from Pharmingen (San Diego, Calif., USA).

Mice

Male BALB/c mice (4-8 weeks old) were obtained from Charles River (Atsugi, Japan) and were maintained in a pathogen-free environment. In some experiments, potassium oxonate (oxonic acid, OA), an uricase inhibitor, was administered with powdered food at a concentration of $5 \% \mathrm{wt} / \mathrm{wt}$ during $8-13$ weeks. Control mice were administered powdered food only for the same periods. All animal procedures were done according to the Guidelines of Laboratory Animal Science of Hiroshima University.

\section{Uric Acid Assay}

Uric acid concentrations in mouse serum samples were determined by a carbonate-phosphotungstate method according to Henry et al. [20].

\section{Contact Hypersensitivity}

Mice were sensitized by application of $50 \mu \mathrm{l}$ of $7 \%$ TNCB in acetone/olive oil $(4: 1)$ to the shaved abdominal skin on day 0 .

Fig. 1. Intradermal injection of MSU augments the TNCB-induced $\mathrm{CHS}$ response. a BALB/c mice were sensitized with $7 \%$ TNCB in the presence or absence of MSU, then challenged 6 days later. Ear thickness was measured at 24-hour intervals (see Materials and Methods). Ear swelling is presented as the mean increase in ear thickness \pm SD over ear thickness before hapten challenge in MSU-treated $(n=5)$ or nontreated mice $(n=6) .{ }^{*} p<0.05$. b Mice were administered OA, an uricase inhibitor, with powdered food at a concentration of 5\% wt/wt before and during the experiments (for 8-13 weeks). Mean body weights \pm SD of OAadministered $(\bigcirc)$ and mock-administered $(O)$ mice from 3 representative experiments $\left(\mathrm{n}=11\right.$ for each group) are shown. ${ }^{*} \mathrm{p}<$ 0.05 . c Serum uric acid concentrations of OA-administered $(\mathrm{n}=$ 4 ; closed bar) and mock-administered ( $\mathrm{n}=3$; open bar) mice after 

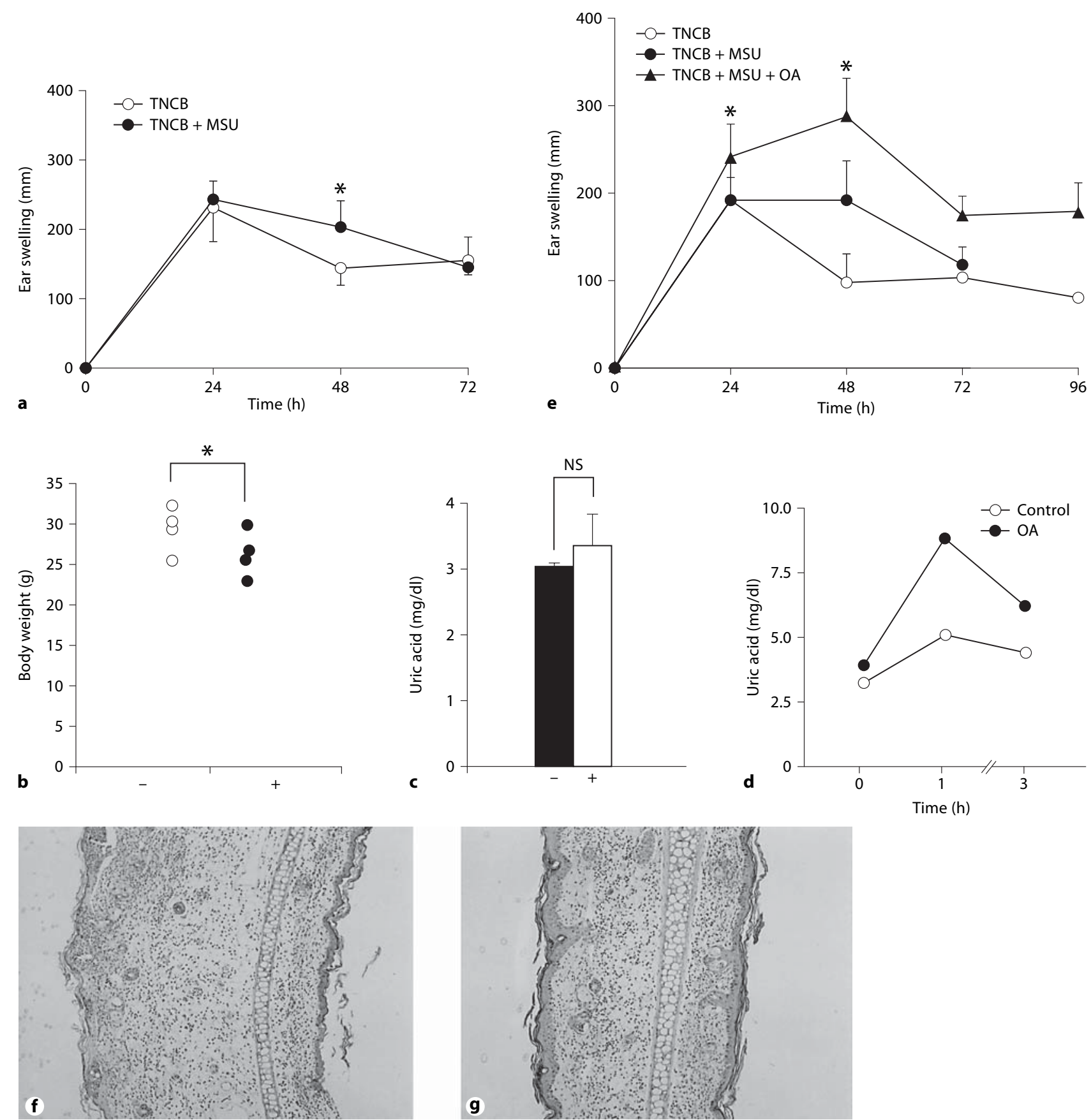

8-11 weeks of administration. NS = Not significant. d OA-administered and mock-administered mice were injected with uric acid solution intraperitoneally at $250 \mathrm{mg} / \mathrm{kg}$ body weight and then serum uric acid concentrations were monitored at 1-hour intervals. Representative results are shown. e The effect of MSU treatment on the CHS response to TNCB in OA-administered mice. OA mice were sensitized to TNCB in the presence of MSU and then challenged as in a. Mice mock administered with powdered food only were sensitized as controls in the presence and the absence of MSU. ${ }^{*} \mathrm{p}<0.05$ for MSU-injected OA mice vs. MSU-injected control mice. $\mathbf{f}, \mathbf{g}$ Histological evaluation of ear samples of MSUadministered OA mice (f) and mock-administered BALB/c mice (g) $48 \mathrm{~h}$ after challenge. Paraffin-embedded sections were stained with hematoxylin and eosin. 
Some mice were injected subcutaneously on the sensitized skin with $2 \mu \mathrm{l}$ of saturated uric acid solution containing $100 \mu \mathrm{g}$ of MSU immediately, $24 \mathrm{~h}$ and $48 \mathrm{~h}$ later. On day $6,10 \mu \mathrm{l}$ of $1 \%$ TNCB in acetone/olive oil (1:9) was applied to both sides (dorsal and ventral) of the right ear. The left ear was treated with vehicle only. The ear thickness of each mouse was measured before and 24, 48, 72 and $96 \mathrm{~h}$ after challenge with an engineer's micrometer (Mitutoyo, Tokyo, Japan).

\section{Histology}

The ears of some mice were harvested $48 \mathrm{~h}$ after challenge and fixed in 10\% PBS-buffered formalin. Paraffin sections were stained with hematoxylin and eosin, and then examined by light microscopy.

\section{Analysis of LN Cells after Sensitization}

On day 0 , mice were sensitized by applying $250 \mu \mathrm{l}$ of $1 \%$ TNCB to the shaved abdominal skin and $15 \mu \mathrm{l}$ to both sides of the ears. Some mice were administered OA and injected with MSU subcutaneously on the sensitized skin immediately, 24, 48 and $72 \mathrm{~h}$ later. Inguinal and axillary LNs from each mouse were pooled, and single-cell suspensions were prepared. LN cells were prestained with anti-CD16/CD32 to block Fc receptors.

For DC analysis, the cells were subsequently stained with anti-CD11c and anti-CD11b and analyzed on an FACS Calibur flow cytometer using CellQuest software (BD Biosciences, San Jose, Calif., USA). We first analyzed, by FITC painting assay, which population in CD11c+ cells of LNs contained skin-derived DCs. We found the CD11c+CD11b+ population largely (97\%) contained FITC+ skin-derived DCs, but the CD11c+CD11bpopulation hardly $(3 \%)$ contained them. Then the cells were also stained with anti-CD86 or anti-CD40, and analyzed for $\mathrm{DC}$ maturation on the $\mathrm{CD} 11 \mathrm{c}+\mathrm{CD} 11 \mathrm{~b}+$ population. In the $\mathrm{CD} 11 \mathrm{c}+\mathrm{CD} 11 \mathrm{~b}-$ population, CD86 mean fluorescence intensity (MFI) was low and not significantly different between the TNCB $+\mathrm{MSU}+\mathrm{OA}$ mice and the TNCB-treated controls. This data indicated that the OA + MSU condition did not affect DCs on peripheral LNs.

For T cell analysis, the cells were prepared $96 \mathrm{~h}$ after sensitization and stained with anti-CD4 and anti-CD8 to analyze T cell subsets or with CD69, CD44 or CD25 to analyze activation. Dead cells were excluded by staining with propidium iodide (SigmaAldrich).

\section{Statistics}

The data were analyzed by the Mann-Whitney U test. $\mathrm{p}<0.05$ were considered significant.

\section{Results}

\section{MSU Augments CHS Responses}

To determine whether MSU modulates immune responses in the skin, we analyzed its effect on CHS in $\mathrm{BALB} / \mathrm{c}$ mice. Groups of mice were sensitized by applying TNCB to shaved abdominal skin and then injecting MSU subcutaneously within the same area. Control mice were similarly sensitized to TNCB and mock injected. Six days later, the mice were rechallenged with TNCB and ear swelling was assessed as a measure of the CHS response. Administration of MSU did not change the maximal ear swelling at $24 \mathrm{~h}$, but the decrease in the ear swelling from 24 to $48 \mathrm{~h}$ was delayed in MSU-administered mice compared to control mice (fig. 1a).

Unlike humans who have mutations in the uricase gene, mice have an active uricase that metabolizes uric acid to allantoin. To prevent degradation of MSU by uricase, mice were administered potassium oxonate (OA mice), an uricase inhibitor, for 8-11 weeks prior to TNCB sensitization [21]. The OA mice appeared healthy, although their average body weight was $17 \%$ lower than that of mock-administered controls (fig. 1b). Serum uric acid concentrations of the OA and control mice revealed no significant difference, indicating that the OA mice did not suffer from hyperuricemia (fig. 1c). However, serum uric acid concentrations were at higher levels for a few hours in the OA mice after intraperitoneal injection of uric acid solution. Clearance of the injected uric acid was less efficient in the OA mice than in the control mice (fig. 1d). To further confirm the effect of MSU on CHS responses, the OA mice were immunized with TNCB in the presence of MSU. MSU significantly increased the CHS response in the OA mice both in magnitude and duration (fig. 1e).

The ears of some mice were harvested $48 \mathrm{~h}$ after challenge and subjected to histological analysis. Edema was pronounced in the ear samples of the OA mice that were TNCB-immunized in the presence of MSU compared with normal mice TNCB-sensitized in the absence of MSU (fig. 1f, g). These observations suggested that MSU augments the CHS response to TNCB.

Fig. 2. MSU enhances the maturation of DCs. a OA mice and mock-administered controls were sensitized to $1 \%$ TNCB in the presence or absence of MSU (see Materials and Methods). Cells from the draining LNs of each mouse were prepared at the indicated times after sensitization, stained with anti-CD11b-FITC, anti-CD11c-APC and anti-CD86-PE and analyzed by flow cytometry. The top left FACS plot shows the expression of CD11b and CD11c on LN cells. Histograms (top right) show the expression of CD86 on CD11b+CD11c+ cells. The bottom left bar graph represents the CD86 MFI \pm SD from MSU-treated OA mice (closed bar) or control mice (open bar) with at least 6 mice per group. CD11b+CD11c+ cells from control mice $48 \mathrm{~h}$ after TNCB sensitization have a bimodal distribution of CD86, while MSU-treated OA mice have a single CD86 high population. The 


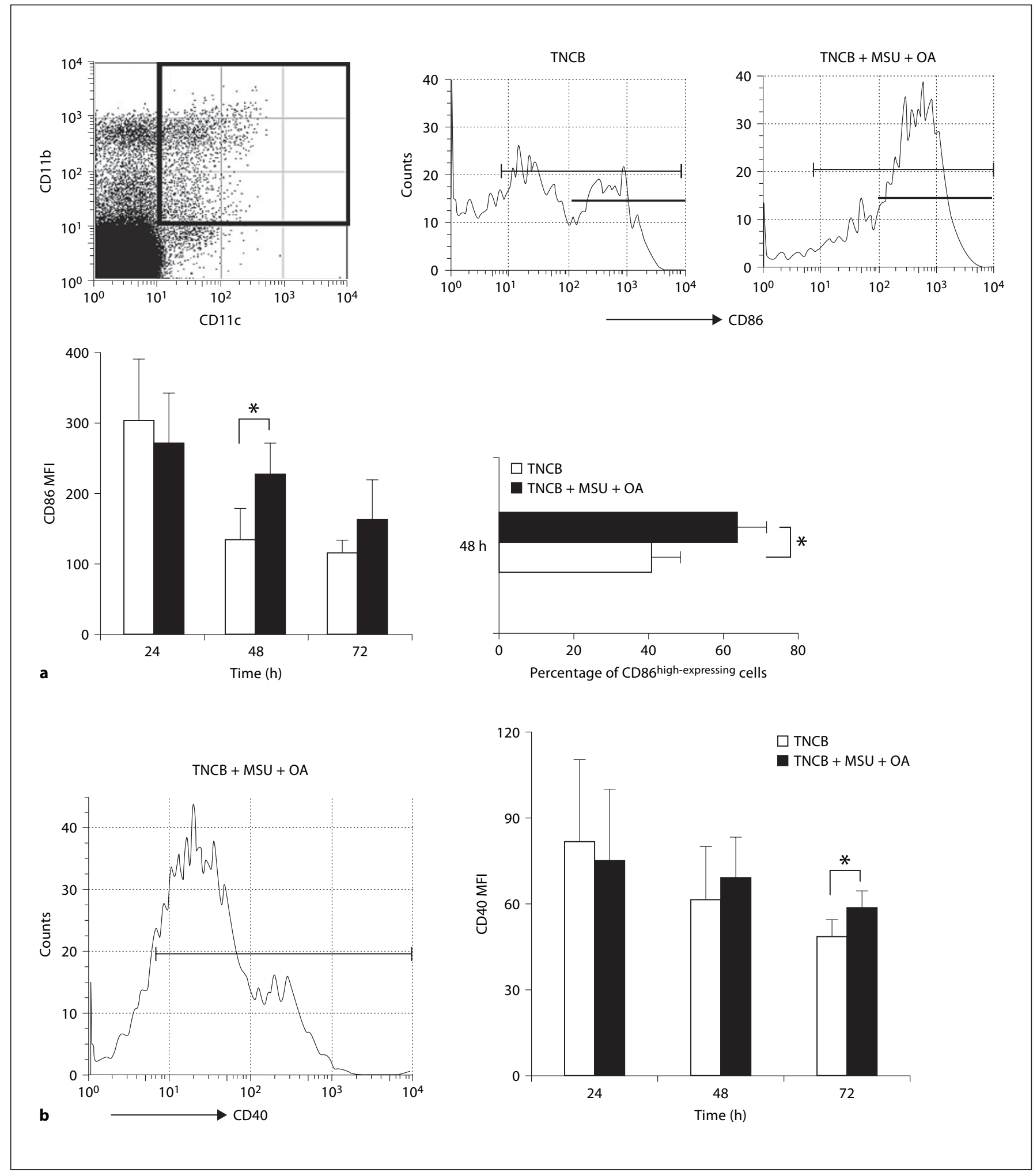

bottom right bar graph shows the frequency of CD86 high-expressing cells, indicated by bold lines in the histograms, from MSU-treated OA mice ( $\mathrm{n}=7$; closed bar) or control mice $(\mathrm{n}=7$; open bar). ${ }^{*} \mathrm{p}<0.05$. b The LN cells were stained with anti-
CD11b-PE, anti-CD11c-APC and anti-CD40-FITC. MFI of $\mathrm{CD} 40+$ cells on $\mathrm{CD} 11 \mathrm{~b}+\mathrm{CD} 11 \mathrm{c}+$ population were analyzed. The bars represent mean MFI \pm SD of the results from at least 5 mice. ${ }^{*} \mathrm{p}<0.05$. 
MSU Enhances the Maturation of DCs in vivo

Shi et al. [13] have shown that MSU drives the maturation of DCs in vitro, leading to an increase in the expression of the costimulatory molecules CD80 and CD86. Therefore, we next analyzed the maturation of DCs in vivo. First, we examined the kinetics of CD86 expression on $\mathrm{CD} 11 \mathrm{~b}+\mathrm{CD} 11 \mathrm{c}+$ cells in the draining LNs of the OA mice sensitized to TNCB in the presence of MSU. The MFI of CD86+ cells was similar in the MSU-administered OA mice and control mice at $24 \mathrm{~h}$ but was enhanced in the MSU-administered OA mice at $48 \mathrm{~h}$ (fig. 2a). In the control mice, a profile of CD86 expression in $\mathrm{CD} 11 \mathrm{~b}+\mathrm{CD} 11 \mathrm{c}+$ cells showed 2 main peaks, representing CD86 high-expressing and CD86 low-expressing cells. However, in MSU-administered OA mice, the CD86 profile showed a single peak of high-expressing cells. The percentage of CD11b+CD11c+ cells expressing high levels of CD86 was higher in MSU-administered OA mice compared with control mice (fig. 2a). The MFI of CD40+ cells was slightly elevated in the MSU-administered OA mice $72 \mathrm{~h}$ after sensitization (fig. $2 \mathrm{~b}$ ).

\section{MSU Enhances Activation of CD4+ and CD8+} T Subsets

The primary effector cells in CHS responses are thought to be CD8+ T cells, whereas CD4+ T cells regulate the magnitude and duration of the response. The MSU danger signal, when delivered with particulate antigen, was shown to prime antigen-specific CD8+ T cells in vivo [13]. Because we found that DCs in the LNs were more activated in MSU-administered OA mice, we then determined whether administration of MSU affected T cells in the draining LNs. The number of CD4+ or CD8+ $\mathrm{T}$ cells was quantitated $96 \mathrm{~h}$ after TNCB immunization with or without MSU administration. MSU had no effect on the number of $\mathrm{T}$ cells in either subset at this time point (fig. 3a). We then analyzed CD69+, CD44+ and CD25+ cells as a measure of activation in these $\mathrm{T}$ cell subsets. The percentage of CD69+ cells was higher in both T cell subsets in the MSU-injected OA mice compared with the mock-injected controls (fig. 3b, c). The percentage of CD44+ cells was slightly elevated in the CD4+ T cell population of MSU-treated OA mice (fig. 3b). The percentage of CD25+ cells was not significantly different in both T cell subsets between MSU-administered OA and control mice, but the MFIs of CD25+ cells in the CD4+CD25+ and $\mathrm{CD} 8+\mathrm{CD} 25+$ cell populations were significantly elevated in MSU-treated OA mice (fig. 3d). These observations suggest that MSU leads to enhanced activation of not only CD8+ T cells, but also CD4+ T cells.

\section{Discussion}

According to the danger hypothesis, injured cells release danger signals that activate APCs and induce the expression of costimulatory molecules. These APCs then prime antigen-specific $\mathrm{T}$ cells. Shi et al. [13] identified uric acid as the principal endogenous danger signal released from injured cells. They showed that uric acid stimulated DC maturation in vitro, increasing the expression of the costimulatory molecules CD86 and CD80. Subsequently, when coinjected with a particulate antigen into mice, uric acid enhanced the generation of a CD8+ $\mathrm{T}$ cell response. In the present study, we have shown that subcutaneous administration of MSU, a crystalline form of uric acid, augments ear swelling response in a mouse CHS model. This observation implies that MSU is a potent endogenous danger signal in the CHS response. By analyzing activation profiles of $\mathrm{CD} 11 \mathrm{c}+\mathrm{CD} 11 \mathrm{~b}+$ cells, and CD4+ or CD8+ cells in the draining LNs, we observed that MSU enhanced DC maturation and increased $\mathrm{T}$ cell activation in vivo, consistent with the observations of Shi et al. [13]. This suggests that the adjuvant effect of MSU occurs in the sensitization phase of the CHS response.

Shi et al. [13] showed that MSU can activate bone marrow-derived DCs in vitro without antigen. Therefore, we raised the question whether MSU can activate DCs by itself or whether it is accessory to the primary contact hypersensitizer in the CHS system. We recently noticed that OA treatment was not necessary to analyze the DC activation by MSU in LNs, although it was necessary to

Fig. 3. Uric acid crystals enhance activation of CD4+ and CD8+ $\mathrm{T}$ subsets. OA mice (closed bars) and control mice (open bars) were treated as in figure 2. Cells from the draining LNs were prepared 4 days after sensitization and stained with anti-CD4-FITC, anti-CD8-APC and anti-CD69-PE or anti-CD44-PE. Alternatively, the cells were stained with anti-CD4-PE, anti-CD8-APC and anti-CD25-FITC. Lymphocytes were first identified by their small size and low granularity, and then analyzed for surface phenotypes. a The bar graph depicts the mean number of CD4+ and CD8+ cells \pm SD from each group of 6 mice. NS $=$ Not significant. b The bar graphs show the mean percentage \pm SD of CD69+, CD44+ or CD25+ cells within the CD4+ or CD8+ T cell subset from 6 MSU-treated OA mice or 6 control mice. ${ }^{* *} \mathrm{p}<0.005$. c FACS plots show representative staining profiles with anti-CD4 vs. anti-CD69 or anti-CD8 vs. anti-CD69. d The bar graph shows the mean MFI \pm SD of CD4+CD25+ cells and of CD8+CD25+ cells from 6 mice each. ${ }^{* *} \mathrm{p}<0.005$. 


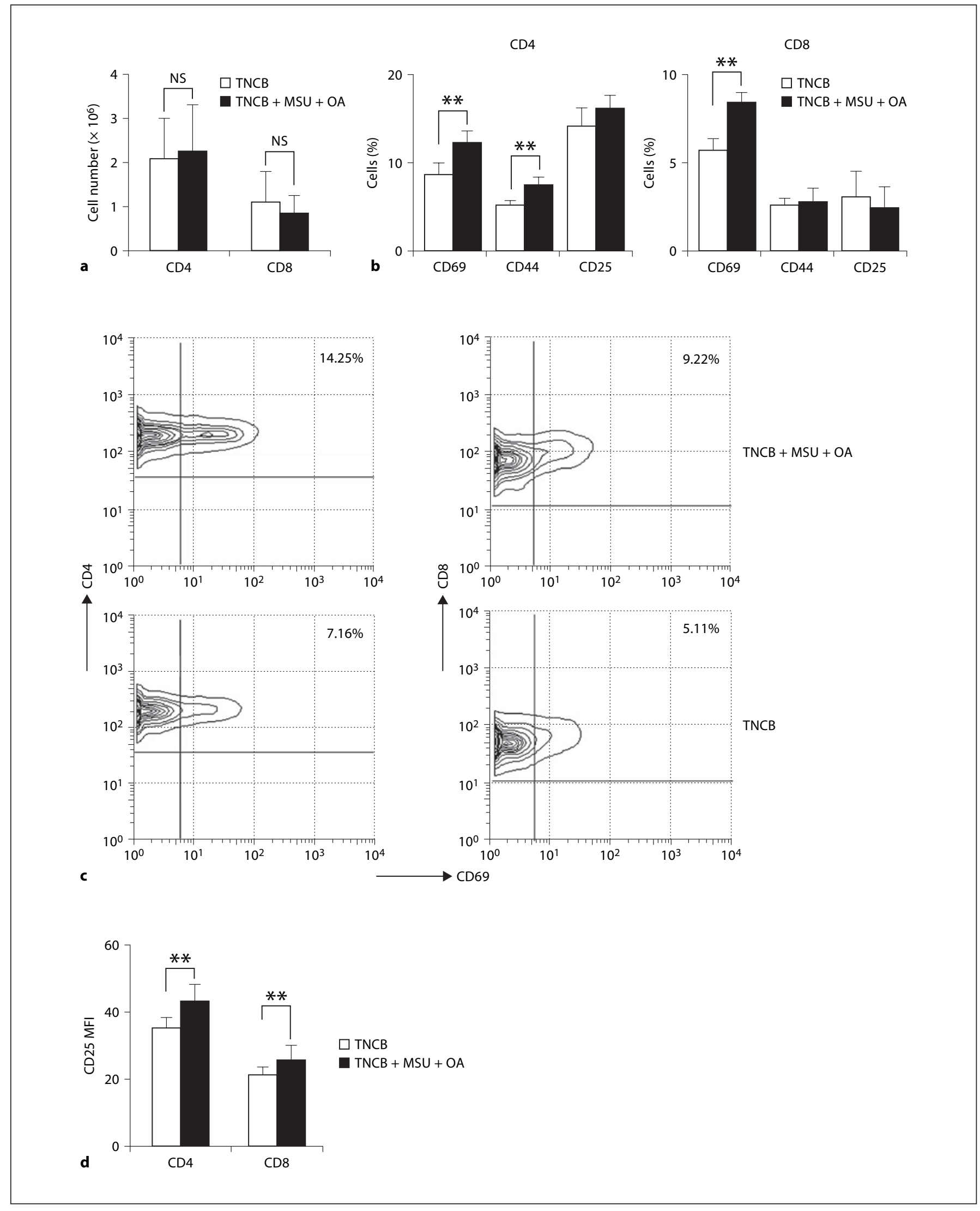


measure the effects on ear swelling. Then, we evaluated the DC activation by measuring CD86 MFI on normal $\mathrm{BALB} / \mathrm{c}$ mice: group 1, TNCB + MSU; group 2, TNCB; group 3, MSU; group 4, no treatment. Comparison of CD86 MFIs between groups 1 and 2 demonstrated statistically significant differences; however, no significant difference was observed between groups 3 and 4 . From these data we propose that MSU is accessory to the primary contact hypersensitizer and cannot activate DCs by itself in the CHS system (data not shown).

Interestingly, our results indicate that the MSU-derived danger signal activates not only CD8+ T cells, but also CD4+ T cells. The primary effector cells in the elicitation phase of CHS responses are currently thought to be CD8+ T cells, whereas CD4+ T cells regulate the magnitude and duration of the response $[22,23]$. Therefore, our observation that MSU enhances CD4 T cell activation implies that MSU can be an endogenous regulator of $\mathrm{CHS}$ response. Further studies are necessary to elucidate the functions of activated CD4+ cells in the elicitation phase.

The innate immune system of mammals has been forged by coevolution with microbes through symbiotic interactions with commensal flora and invading pathogens. Because of both the beneficial and harmful relationships with microbes, mammals have developed an elaborate sensing network to microbes. A recent growing number of non-Toll-like innate immune receptors, including Nod1, Nod2, NALP3, Ipaf and Naip, are recognized as part of this sensing network [2]. The intracellular Nod-like receptors are a family of sensors that respond to microbial motifs and danger signals and are emerging as critical components of innate immune responses and inflammatory response in mammals. Recent reports have shown that the NALP3 inflammasome is essential for a CHS response to hapten trinitrophenyl at the sensitization phase [17]. In addition, the release of MSU from dying cells associated with gout can act as a danger signal to activate the NALP3 inflammasome [16]. Taken together with our observations, this implies that MSU released from injured cells and the antigenic signal derived from haptens in CHS may share a common Nod-like receptor that acts as a platform to synergize and activate the inflammatory response. Therefore, we propose that NALP3 inflammasome is a common platform for these signals, explaining why MSU could exacerbate CHS in our mouse model.

From a clinical perspective, our observations have several implications for allergic skin diseases. CHS is a clinically important syndrome known as allergic contact dermatitis. In allergic contact dermatitis, 3 main pathological changes are seen: skin barrier disruption, epidermal cellular changes and cytokine release. These pathological changes may be associated with the release of danger signals, including MSU. Smith et al. [24] proposed that irritancy plays a crucial role in the development of allergic contact dermatitis. Consistent with this hypothesis, we propose that skin damage could play a crucial role in the development and deterioration of allergic skin diseases.

\section{Acknowledgements}

This study was supported by Health and Labor Sciences Research Grants (Research on Allergic Disease and Immunology). We thank M. Miyazaki for helpful discussions.

\section{References}

$>1$ Kawai T, Akira S: Pathogen recognition with Toll-like receptors. Curr Opin Immunol 2005;17:338-344.

$>2$ Fritz JH, Ferrero RL, Philpott DJ, Girardin SE: Nod-like proteins in immunity, inflammation and disease. Nat Immunol 2006;7: 1250-1257.

$>3$ Seong SY, Matzinger P: Hydrophobicity: an ancient damage-associated molecular pattern that initiates innate immune responses. Nat Rev Immunol 2004;4:469-478.

$\checkmark 4$ Matzinger P: Tolerance, danger, and the extended family. Annu Rev Immunol 1994;12: 991-1045.

5 Gallucci S, Lolkema M, Matzinger P: Natural adjuvants: endogenous activators of dendritic cells. Nat Med 1999;5:1249-1255.
-6 Gallucci S, Matzinger P: Danger signals: SOS to the immune system. Curr Opin Immunol 2001;13:114-119.

7 Matzinger P: The danger model: a renewed sense of self. Science 2002;296:301-305.

$>8$ Pulendran B: Immune activation: death, danger and dendritic cells. Curr Biol 2004; 14:R30-R32.

$\checkmark 9$ Matzinger P: Friendly and dangerous signals: is the tissue in control? Nat Immunol 2007;8:11-13.

10 Srivastava P: Interaction of heat shock proteins with peptides and antigen presenting cells: chaperoning of the innate and adaptive immune responses. Annu Rev Immunol 2002;20:395-425.
11 Coutinho-Silva R, Persechini PM, Bisaggio RD, Perfettini JL, Neto AC, Kanellopoulos JM, Motta-Ly I, Dautry-Varsat A, Ojcius DM: P2Z/P2X7 receptor-dependent apoptosis of dendritic cells. Am J Physiol 1999;276: C1139-C1147.

12 Shi Y, Zheng W, Rock KL: Cell injury releases endogenous adjuvants that stimulate cytotoxic T cell responses. Proc Natl Acad Sci USA 2000;97:14590-14595.

13 Shi Y, Evans JE, Rock KL: Molecular identification of a danger signal that alerts the immune system to dying cells. Nature 2003; 425:516-521.

$14 \mathrm{Hu}$ DE, Moore AM, Thomsen LL, Brindle KM: Uric acid promotes tumor immune rejection. Cancer Res 2004;64:5059-5062. 
15 Shi Y, Galusha SA, Rock KL: Elimination of an endogenous adjuvant reduces the activation of CD8 T lymphocytes to transplanted cells and in an autoimmune diabetes model. J Immunol 2006;176:3905-3908.

16 Martinon F, Petrilli V, Mayor A, Tardivel A, Tschopp J: Gout-associated uric acid crystals activate the NALP3 inflammasome. Nature 2006;440:237-241.

17 Sutterwala FS, Ogura Y, Szczepanik M, LaraTejero M, Lichtenberger GS, Grant EP, Bertin J, Coyle AJ, Galan JE, Askenase PW, Flavell RA: Critical role for NALP3/CIAS1/Cryopyrin in innate and adaptive immunity through its regulation of caspase-1. Immunity 2006;24:317-327.
18 Grabbe S, Schwarz T: Immunoregulatory mechanisms involved in elicitation of allergic contact hypersensitivity. Immunol Today 1998;19:37-44.

19 Cavani A, Albanesi C, Traidl C, Sebastiani S, Girolomoni G: Effector and regulatory $\mathrm{T}$ cells in allergic contact dermatitis. Trends Immunol 2001;22:118-120.

20 Henry RJ, Sobel C, Kim J: A modified carbonate-phosphotungstate method for the determination of uric acid and comparison with the spectrophotometric uricase method. Am J Clin Pasthol 1957;28:157-160.

21 Stavric B, Nera EA: Use of the uricase-inhibited rat as an animal model in toxicology. Clin Toxicol 1978;13:47-74.
22 Wang B, Fujisawa H, Zhuang L, Freed I, Howell BG, Shahid S, Shivji GM, Mak TW, Sauder DN: CD4+ Th1 and CD8+ type $1 \mathrm{cy}$ totoxic $\mathrm{T}$ cells both play a crucial role in the full development of contact hypersensitivity. J Immunol 2000;165:6783-6790.

23 He D, Wu L, Kim HK, Li H, Elmets CA, Xu $\mathrm{H}$ : CD8+ IL-17-producing T cells are important in effector functions for the elicitation of contact hypersensitivity responses. J Immunol 2006; 177:6852-6858.

24 Smith HR, Basketter DA, McFadden JP: Irritant dermatitis, irritancy and its role in allergic contact dermatitis. Clin Exp Dermatol 2002;7:138-146. 